\title{
Invitation a soumettre des communications, Congrès annuel de l'ASCP, 1984
}

L'Association invite ses membres à soumettre des sujets de communications à présenter lors du Congrès annuel de 1984 de l'Association canadienne de science politique qui aura lieu à l'Université de Guelph, Guelph, Ontario, 10, 11, et 12 juin 1984.

Une proposition devrait comporter le titre, une résumé (environ 300 mots) de la thèse de la communication, une explication de la méthodologie employée et l'adresse de l'auteur ou les adresses des co-auteurs.

On pourrait soumettre un sujet au membre du Comité responsable du domaine en question. Le Comité recevra avec plaisir toute proposition relative au programme, telle thèmes pour les sessions plénières. Les sujets qui ne correspondent à aucune section peuvent être soumis au président du programme. 1983.

La date limite pour des sıjets dı Comité du programme sera le 4 novembre

Les communications complètes ne doivent pas excéder 30 pages de longueur, à double interligne et ne doivent pas avoir été publiées auparavant.

Les auteurs doivent envoyer deux exemplaires de leurs communications au secrétariat de l'Association avant le ler mai et avoir 100 exemplaires disponibles pour distribution aux membres.

Président du Comité du programme: Michael Atkinson, Department of Political Science, McMaster University, Hamilton, Ontario LSS $4 M 4$

Vice-président: (et président 1985) André Bernard, Département de science politique, Université du Québec à Montréal, Montréal, P.Q. H3C $3 E 9$

Représantant local: Pat Kyba, Department of Political Studies, University of Guelph, Guelph, Ontario NIG $2 W I$

Section

Politique canadienne

Politique provinciale

Politique publique, administration publique et droit publique comportement politique

Idéologie politique et analyse politique
Sociologie politique et

Chef de section

André Bernard, Département de science politique, Université du Québec à Montréal, P.Q. H3C 3E9

Geoff Weller, Department of Political Science, Lakehead University, Thunder Bay, Ontario P7B 5EI

Carolyn Tuohy, Institute for Policy Analysis, University of Toronto, Toronto, Ontario M5S 1Al

Richard Price, Department of Political Science, University of Windsor, Windsor, Ontario N9B 3P4

Tom Lewis, Department of Political Science, McMaster University, Hamilton, Ontario L8S 4M4

Les communications touchant "l'analyse politique et l'histoire „ doivent être soumises à cette section. Si nécessaire, deux séries de sessions seront mises sur pied.

Politique comparée: Systèmes de l'ouest

Politique comparée:

Steven Bornstein, Department of Political Science, McGill University, Montreal, P.Q. H3A 2T7

William Graf, Department of Political Studies, University of Systèmes du Tiers-monde Guelph, Guelph, Ontario N1G 2W1 et systèmes socialistes

L'économie politique

Rianne Mahon, School of Public Administration, Carleton University, Ottawa, Canada KIS 5B6

Relations internationales John Kirton, Department of Political Science, University of Toronto, Toronto, Ontario MSS IAI

Politique locale et urbaine Andrew Sancton, Department of Political Science, University of Westem Ontario, London, Ontario N6A 5C2

Sessions plénières et spéciales
Geoff Weller, Department of Political Science, Lakehead University, Thunder Bay, Ontario P7B 5E1 